01

\title{
Многомасштабный анализ ритмических процессов с меняющимися во времени характеристиками
}

\author{
(C) Н.М. Куприяшкина, О.Н. Павлова, А.Н. Павлов \\ Саратовский национальный исследовательский государственный университет им. Н.Г. Чернышевского, Саратов, Россия \\ ฯ E-mail: pavlov.alexeyn@gmail.com
}

Поступило в Редакцию 23 января 2020г.

В окончательной редакции 7 июня 2020г.

Принято к публикации 7 июня 2020 г.

\begin{abstract}
Рассматривается проблема диагностики структурных изменений нестационарных процессов в случае зависимости степени нестационарности от частотного диапазона. На примере динамики медленных волн сна проводится многомасштабный анализ экспериментальных данных и обсуждается возможность улучшения качества диагностики путем выбора подходящего базиса вейвлет-функций.
\end{abstract}

Ключевые слова: нестационарный процесс, вейвлет-анализ, ритмическая динамика.

DOI: 10.21883/PJTF.2020.18.49992.18219

В динамике многих систем нестационарность ассоциируется с низкочастотным трендом, устранение которого посредством фильтра верхних частот позволяет применять к отфильтрованным данным классический инструментарий методов цифровой обработки сигналов [1]. Но есть и более сложные ситуации, когда наряду с изменениями локального среднего уровня нестационарность связана с вариациями характеристик ритмических процессов в некотором диапазоне частот [2-6]. При исследовании этого диапазона необходимо либо использовать методы анализа систем с меняющимися во времени параметрами, либо проводить оценки характеристик режима функционирования системы по малому объему выборки в предположении квазистационарности динамики на небольших интервалах времени. Широко используемым инструментарием решения таких задач является вейвлет-анализ [7]. При проведении цифровой обработки больших объемов экспериментальных данных применяются быстрые алгоритмы многомасштабного (или кратномасштабного) анализа, предусматривающие разложение по ортонормированному базису функций, например вейвлетам семейства Добеши[7]. Правильный выбор базиса может иметь важное значение, поскольку это позволяет эффективно решать многие практические задачи [8-12], однако большое разнообразие вейвлетфункций делает выбор в значительной степени субъективным.

В настоящей работе обсуждается проблема диагностики структурных изменений нестационарных процессов для случая, когда степень нестационарности зависит от частотного диапазона. На примере динамики медленных волн сна мы проводим многомасштабный анализ экспериментальных данных и показываем, как качество диагностики может быть улучшено за счет выбора подходящего базиса вейвлет-функций.

Многомасштабный анализ предусматривает процедуру пирамидального разложения сигнала с помощью набора зеркальных фильтров, которые формируются при масштабных преобразованиях и смещениях скейлингфункции $\phi(t)$ и вейвлета $\psi(t)$ :

$$
\phi_{j, k}=2^{j / 2} \phi\left(2^{j} t-k\right), \quad \psi_{j, k}=2^{j / 2} \psi\left(2^{j} t-k\right) .
$$

Разложение проводится в базисе вейвлетов Добеши [7]. На произвольном уровне разрешения $m$ анализируемый сигнал представляется в виде

$$
x(t)=\sum_{k} s_{m, k} \phi_{m, k}(t)+\sum_{j \geqslant m} \sum_{k} d_{j, k} \psi_{j, k}(t),
$$

где $s_{m, k}$ и $d_{j, k}-$ коэффициенты аппроксимации и детализации. С учетом компактности носителя вейвлетов Добеши переменная $t$ принимает значения в пределах вейвлет-функции, область задания которой зависит от масштаба. Вариабельность коэффициентов детализации на разных масштабах $j$, характеризуемая дисперсией

$$
\sigma(j)=\sqrt{\frac{1}{M} \sum_{k=0}^{M-1}\left[d_{j, k}-\left\langle d_{j, k}\right\rangle\right]^{2}},
$$

используется для диагностики структурных изменений сигналов [13]. Здесь $M-$ число коэффициентов детализации на масштабе $j$, которое определяет диапазон изменения индекса $k$, угловыми скобками обозначено усреднение по всем коэффициентам детализации на данном масштабе.

Рассмотрим в качестве примера задачу диагностики изменений структуры сигнала электрической активности мозга во время сна. Соответствующие изменения связаны с динамикой медленных волн сна $(0.5-4 \mathrm{~Hz})$, исследование которых вызывает значительный интерес в последние годы $[14,15]$. Известно, что в течение ночи у человека наблюдается четкая последовательность сменяющих друг друга фаз сна, которая отличается при возрастных изменениях или при патологии $[16,17]$. Но, 


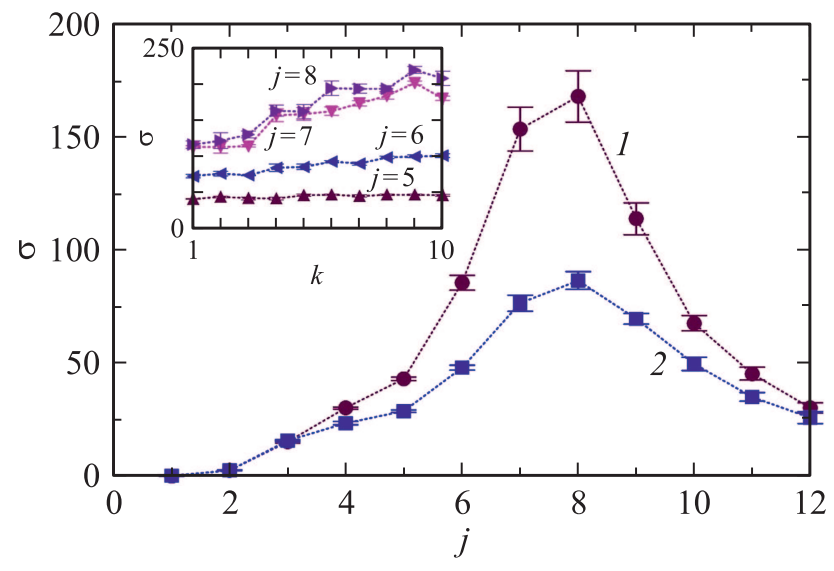

Рис. 1. Зависимости дисперсии коэффициентов детализации от масштаба для фазы медленного (1) и быстрого (2) сна для вейвлета $D_{s}^{8}$. Усреднение осуществлялось по сегментам ЭЭГ длительностью $2 \mathrm{~min}$. На вставке приведены значения дисперсии в зависимости от номера сегмента, демонстрирующие нестационарный характер для масштабов $j=7$ и 8 .

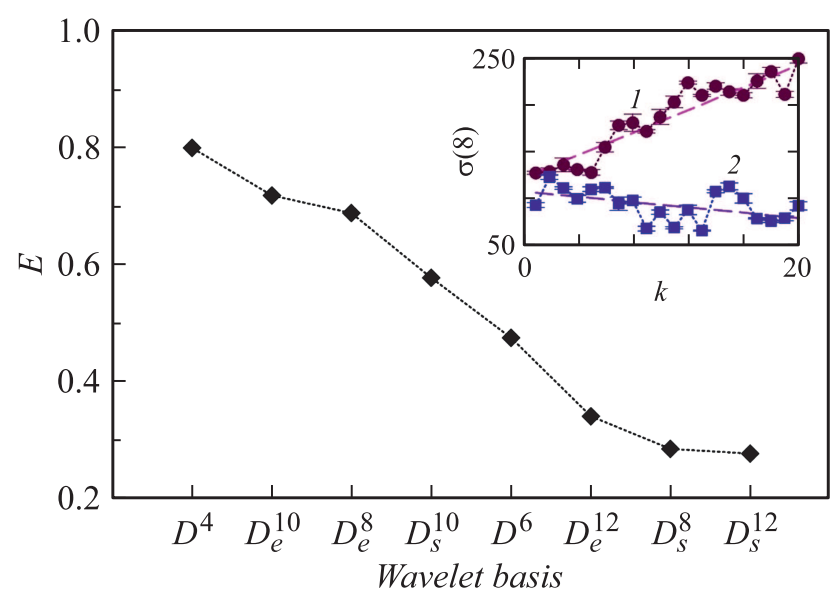

Рис. 2. Величина отклонения от усредненного значения $\sigma(j)$, нормированного в диапазоне от 0 до 1 и усредненного по уровням разложения $j$. На вставке приведены значения дисперсии $\sigma(8)$ для уровня $j=8$ в зависимости от номера сегмента длительностью $1 \mathrm{~min}$, демонстрирующие различное поведение для фазы медленного (1) и быстрого (2) сна.

несмотря на существование таких отличий, они до сих пор мало изучались.

В проводимых исследованиях проанализированы изменения во времени характеристик сигналов электроэнцефалограмм (ЭЭГ) семи здоровых людей во время разных фаз сна. При частоте дискретизации сигналов $250 \mathrm{~Hz}$ масштабы $j$, соответствующие медленным волнам сна, находятся в диапазоне от 6 до 9. Именно в этом диапазоне наблюдаются максимальные различия для фаз медленного и быстрого сна (рис. 1, зависимости 1 и 2). При этом, однако, обнаруживается следующий эффект: изменение характеристик медленных волн в пределах одной фазы сна (зависимости для $j=7$ и 8 на вставке к рис. 1), которое сопровождается приближенно постоянными значениями дисперсии вейвлет-коэффициентов для более высокочастотных ритмов (зависимость для $j=5)$. Этот эффект представляет интерес с точки зрения задач диагностики, так как в рассматриваемом примере для фазы быстрого сна изменение дисперсии во времени носит другой характер (вставка на рис. 2), и можно предположить, что характер соответствующих зависимостей различается также для нормы и патологии.

Важным аспектом для практического применения рассматриваемого подхода является выбор вейвлет-базиса. Несмотря на то что разные вейвлеты семейства Добеши приводят к качественно похожим результатам, оптимальный выбор базиса позволит снизить разброс характеристик, вычисляемых по фрагментам экспериментальных данных, и тем самым повысить надежность разделения сигналов. В проводимых расчетах мы выбирали разную область задания вейвлета, включая функции семейства Добеши с экстремальной фазой (обозначены символом $e$ на рис. 2) и наименее асимметричные вейвлеты $(s)$. Проводился поиск вейвлета, обеспечивающего минимальный разброс оцениваемых характеристик (3) по сравнению со значениями, которые получаются при усреднении расчетов по выбранным базисам. С учетом того, что коэффициенты детализации меняются, оценки проводились следующим образом: для каждого вейвлета вычислялось среднее по модулю отклонение $E$ от усредненного значения $\sigma(j)$, и оно нормировалось в диапазоне от 0 до 1. Затем проводилось усреднение по уровням разложения $j$. Полученные результаты приведены на рис. 2 в порядке снижения величины $E$. В соответствии с рис. 2 максимальный разброс результатов наблюдается для вейвлета $D^{4}$, обладающего наименьшей областью задания. Однако увеличение области задания (и соответственно повышение гладкости вейвлет-функции) не всегда улучшает ситуацию. Высокие значения $E$ чаще наблюдались для асимметричных вейвлет-функций $\left(D_{e}^{10}, D_{e}^{8}\right)$, тогда как уменьшение разброса оцениваемых характеристик (3) происходило при выборе наименее асимметричных вейвлетов $\left(D_{s}^{8}, D_{s}^{12}\right)$. Именно эти функции представляются наиболее подходящими для рассматриваемой задачи по результатам сравнительного анализа для всех испытуемых. По аналогии с медленными волнами сна, выбранными в качестве иллюстративного примера, анализ процессов с нестационарной динамикой в некотором диапазоне частот может проводиться для различных технических систем.

\section{Благодарности}

Авторы выражают признательность О.В. Иванову за предоставление 64-разрядных коэффициентов вейвлет-фильтров семейства Добеши и полезные обсуждения. 


\section{Финансирование работы}

Исследование выполнено при поддержке гранта Правительства РФ (№ 075-15-2019-1885). А.Н.П. благодарит за поддержку в рамках гранта Президента РФ для государственной поддержки ведущих научных школ РФ (НШ-2594.2020.2), а также математический центр СГУ им. Н.Г. Чернышевского.

\section{Соблюдение этических стандартов}

Все процедуры, выполненные в исследовании с участием людей, соответствуют этическим стандартам институционального и национального комитета по исследовательской этике и Хельсинкской декларации 1964 г. и ее последующим изменениям или сопоставимым нормам этики. От каждого из включенных в исследование участников было получено информированное добровольное согласие.

\section{Конфликт интересов}

Авторы заявляют, что у них нет конфликта интересов.

\section{Список литературы}

[1] Bendat J.S., Piersol A.G. Random data: analysis and measurement procedures. 4th ed. New Delhi: Wiley India Pvt., Ltd, 2013. 620 p.

[2] Bernaola-Galvan P., Ivanov P.Ch., Amaral L.A.N., Stanley H.E. // Phys. Rev. Lett. 2001. V. 87. P. 168105.

[3] Jennings H.D., Ivanov P.Ch., Martins A.M., da Silva P.C., Vishwanathan G.M. // Physica A. 2004. V. 336. P. 585-594.

[4] Короновский А.А., Макаров В.А., Павлов А.Н., Ситникова Е.Ю., Храмов А.Е. Вейвлеты в нейродинамике и нейрофизиологии. М.: Физматлит, 2013. 271 с.

[5] Schulte J.A. // Nonlin. Process. Geophys. 2016. V. 23. P. $257-$ 267.

[6] Frolov N.S., Grubov V.V., Maksimenko V.A., Lüttjohann A., Makarov V.V., Pavlov A.N., Sitnikova E., Pisarchik A.N., Kurths J., Hramov A.E. // Sci. Rep. 2019. V. 9. P. 7243.

[7] Daubechies I. Ten lectures on wavelets. Philadelphia: S.I.A.M., 1992. $378 \mathrm{p}$.

[8] Грубов В.В., Руннова А.Е., Короновский А.А., Храмов А.Е. // Письма в ЖТФ. 2017. Т. 43. В. 13. С. 58-64.

[9] Божсокин С.В., Жарко С.В., Ларионов Н.В., Литвинов А.Н., Соколов И.М. // ЖТФ. 2017. Т. 87. В. 6. С. 822 830.

[10] Божокин С.В., Соколов И.М. // ЖТФ. 2018. Т. 88. В. 12. C. $1771-1778$.

[11] Павлова О.Н., Павлов А.Н. // Письма в ЖТФ. 2018. Т. 44. B. 14. C. $80-85$.

[12] Гришина Д.С., Павлов А.Н., Павлова О.Н., Руннова А.Е. // Письма в ЖТФ. 2019. Т. 45. В. 16. С. 24-26.

[13] Thurner S., Feurstein M.C., Teich M.C. // Phys. Rev. Lett. 1998. V. 80. P. 1544-1547.

[14] Bresch E., Großekathöfer U., Garcia-Molina G. // Front. Comput. Neurosci. 2018. V. 12. P. 85.
[15] Garcia-Molina G., Tsoneva T., Jasko J., Steele B., Aquino A., Baher K., Pastoor S., Pfundtner S., Ostrowski L., Miller B., Papas N., Riedner B., Tononi G., White D.P. // J. Neural Eng. 2018. V. 15. P. 066018.

[16] Bartsch R.P., Schumann A.Y., Kantelhardt J.W., Penzel T., Ivanov P.Ch. // PNAS. 2012. V. 109. P. 10181-10186.

[17] Liu K.K.L., Bartsch R.P., Lin A., Mantegna R.N., Ivanov P.Ch. // Front. Neural Circuits. 2015. V. 9. P. 62. 